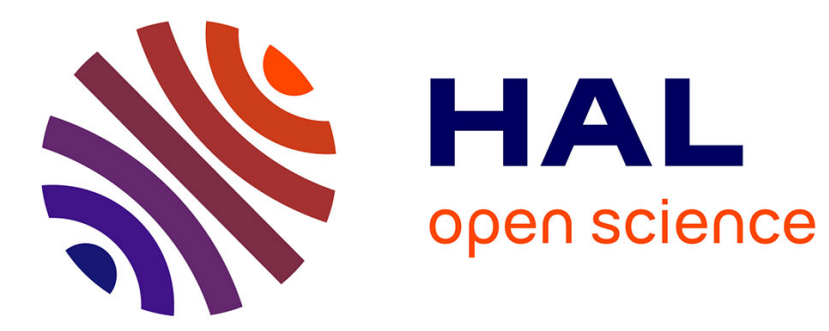

\title{
La société de secours mutuels des Vrais Amis (1820-1898) : comment les bouchers parisiens ont-ils organisé leur protection sociale au XIXe siècle? \\ Sylvain Leteux
}

\section{- To cite this version:}

Sylvain Leteux. La société de secours mutuels des Vrais Amis (1820-1898): comment les bouchers parisiens ont-ils organisé leur protection sociale au XIXe siècle?. RHPS - Revue d'histoire de la protection sociale, 2010, 3, pp.9-27. halshs-00796037

\section{HAL Id: halshs-00796037 \\ https://shs.hal.science/halshs-00796037}

Submitted on 1 Mar 2013

HAL is a multi-disciplinary open access archive for the deposit and dissemination of scientific research documents, whether they are published or not. The documents may come from teaching and research institutions in France or abroad, or from public or private research centers.
L'archive ouverte pluridisciplinaire HAL, est destinée au dépôt et à la diffusion de documents scientifiques de niveau recherche, publiés ou non, émanant des établissements d'enseignement et de recherche français ou étrangers, des laboratoires publics ou privés. 


\title{
La société de secours mutuels des Vrais Amis (1820-1898) : comment les bouchers parisiens ont-ils organisé leur protection sociale au XIX ${ }^{\mathrm{e}}$ siècle?
}

\author{
Sylvain Leteux, post-doctorant (IRHIS, Lille 3)
}

Les travailleurs ont souvent eu le souci de leur protection sociale, c'est-à-dire la prévoyance des risques liés à l'incapacité de travail, qu'il s'agisse de la maladie, de l'infirmité ou de la vieillesse. Sous l'Ancien Régime, l'appartenance à une confrérie apportait à la plupart des artisans une certaine solidarité professionnelle - à forte connotation religieuse - qui venait compléter l'entraide familiale, notamment pour bénéficier d'obsèques dignes. Depuis 1945, avec la mise en place de l'Etat-Providence et la généralisation des systèmes obligatoires de protection sociale (pour la maladie, la vieillesse et le chômage), les salariés français bénéficient d'un minimum garanti de protection sociale. La "protection sociale » du travailleur est moins connue et plus complexe à saisir avant la loi du $1^{\text {er }}$ avril 1898, qui constitue la «Charte» de la mutualité moderne et marque «l'entrée de la société française dans l'ère de l'assurance sociale $»^{1}$. Avec la loi Le Chapelier du 14 juin 1791, la Révolution française a interdit toute association professionnelle, pour empêcher les coalitions mais aussi les sociétés de secours, « au motif que l'assistance relève désormais de la responsabilité étatique $»^{2}$. La période du XIXe siècle est intéressante à étudier pour comprendre comment un groupe professionnel, les bouchers parisiens, ont mis en place une société de secours mutuels leur garantissant un minimum de protection sociale, à une époque réputée « libérale », où l'Etat n'a pas vocation à prendre en charge ce type de compétence. Une source riche, l'ouvrage de Louis Goyard, ancien trésorier de la société des Vrais Amis, publié en 1887-1888, nous permet de retracer avec détail l'histoire du mutualisme chez les bouchers de Paris ${ }^{3}$. L'approche sera chronologique car il s'agit avant tout de comparer le fonctionnement d'un mutualisme original au début du XIX ${ }^{\mathrm{e}}$ dans un cadre très particulier, hors-norme et hyper-protégé à cause du système néo-corporatif dont bénéficie la boucherie parisienne entre 1811 et 1858, avec une situation « normalisée » dans la seconde moitié du siècle4.

${ }^{1}$ Gibaud Bernard, Mutualité, assurances (1850-1914) : les enjeux, Paris, Economica, 1998, p. IX.

${ }^{2}$ Ibid., p. 13.

${ }^{3}$ Goyard Louis, "Origine et développement des sociétés de secours mutuels », Bulletin mensuel de la Société de prévoyance et de secours mutuel de la boucherie de Paris (les V rais Amis), 1887-1888. BNF, 4 R 916.

${ }^{4}$ Ce particularisme néo-corporatif du commerce de la boucherie à Paris entre 1811 et 1858 est l'un des thèmes centraux de ma thèse de doctorat, Libéralisme et corporatisme chez les bouchers parisiens (1776-1944), soutenue en 2005 à Lille 3. 


\section{Naissance de deux sociétés de secours mutuels: rivales ou jumelles?}

Entre 1791 et 1830, 193 sociétés de secours mutuels sont créées à Paris ${ }^{5}$. On distingue généralement deux types de sociétés de secours mutuels au début du XIX ${ }^{\mathrm{e}}$ siècle : la mutualité de secours, organisant l'entraide contre les aléas de la vie sous le contrôle des autorités publiques et patronales, et la mutualité ouvrière de prévoyance et de résistance qui concerne plutôt les métiers qualifiés dans les grandes villes. Les deux sociétés de secours mutuels créées par les bouchers parisiens, en 1820 et en 1824, relèvent plutôt de la première catégorie, mais la frontière n'est pas étanche entre les deux types de sociétés. Le rythme de création des sociétés de secours mutuels s'accélère alors en France: 180 sous l'Empire, 475 sous la Restauration, 1295 pendant la monarchie de Juillet. Mais, comme le souligne Bernard Gibaud, «le processus de mutualisation demeure limité, pendant la première moitié du XIX siècle, à une frange minoritaire de la population ouvrière urbaine $»^{6}$. Michel Dreyfus précise qu'en 1823, « on compte à Paris 160 sociétés: la grande majorité d'entre elles - 132 totalisant 11343 membres - est formée par des ouvriers d'une même industrie »7.

La société de secours mutuels des Vrais Amis est fondée en 1820 par quatre garçons bouchers, le projet ayant été lancé le 24 décembre 1819 par Pierre Taffoureau, étalier maitre garçon. La dimension religieuse sous-entendue par cette date du 24 décembre se retrouve dans la devise proposée par Taffoureau : «Aimons-nous les uns les autres. Aidons-nous les uns les autres. » Les autres devises de la société ne présentent pas de connotation religieuse : «Prévoyance, Humanité, Sagesse » et « En pensant à notre avenir, l'aspect de nos vieux jours ne nous fait pas frémir ». Dans un article de 1983, Pierre Bony, directeur général honoraire de la Caisse nationale d'assurance vieillesse des industries et commerçants, reprend à son compte le lien de cause à effet suggéré en 1910 par Henry Matrot entre l'ouverture des cinq grands abattoirs publics à Paris en 1818 et l'apparition des Vrais Amis ${ }^{8}$. Dans ses Vieux Souvenirs, Henry Matrot indique que, suite à la décision de fermer toutes les tueries particulières situées à Paris et d'obliger les bouchers à abattre dans les abattoirs publics, « tous les garçons bouchers de Paris se trouvèrent réunis dans cinq grands ateliers, de sorte qu'ils apprirent à se connaittre plus intimement que par le passé en raison de leurs relations journalières $»^{9}$. Cette explication est recevable mais je préfère celle donnée par Louis

5 Bennet Jean, La mutualité à travers sept siècles d'Histoire, Paris, Coopérative d'information et d'édition mutualiste, 1975, p. 191.

${ }^{6}$ Gibaud Bernard, op. cit., p. 19.

7 Dreyfus Michel, Liberté, égalité, mutualité: mutualisme et syndicalisme 1852-1967, Paris, Editions ouvrières, 2001, p. 25.

8 Bony Pierre, «Une doyenne : la société mutualiste des bouchers de Paris, les Vrais Amis », Colloque sur l'Histoire de la Sécurité sociale : actes du 108 e congrès national des sociétés savantes, Grenoble 1983, Paris, Association pour l'étude de l'Histoire de la Sécurité sociale, 1984, p. 13.

${ }^{9}$ Matrot Henry, Vieux Sowvenirs sur les associations syndicales et mutuelles et les anciennes pratiques professionnelles de la corporation de la boucherie, Montrouge, Imprimerie Multigraphica, 1935, p. 40. Ancien chevillard, Henry 
Goyard, qui inscrit la fondation des Vrais Amis dans la volonté de retrouver les structures d'entraide, confraternelles ou corporatives, de l'Ancien Régime, détruites pendant la Révolution (le compagnonnage n'a jamais concerné les bouchers). Son choix de citer un texte de 1852 d'Hubbard est d'ailleurs très révélateur: «Lorsque l'Assemblée Constituante (...) eut supprimé les maitrises et jurandes, l'ouvrier se trouva livré sans défense à tous les accidents d'une vie isolée, et condamné ainsi à lutter seul contre les maladies, le chômage et la vieillesse $»^{10}$.

La société des Vrais Amis est reconnue le 15 mars 1820 par le préfet de police de Paris. La première réunion des Vrais Amis se tient le 20 mars 1820 : sur les 45 personnes présentes, 20 sont admises dans la mutuelle. Le droit d'admission est de cent francs et la cotisation d'un franc par semaine, avec un système de pénalités de retard quand le nouveau membre a plus de 30 ans. Le conseil d'administration est formé en 1820, avec Caron comme président, Taffoureau comme trésorier et trois administrateurs. Les prestations proposées aux adhérents sont les suivantes : payer une indemnité de 2,50 F par jour en cas de maladie, une indemnité de $2 \mathrm{~F}$ par jour de convalescence, une pension d'1 F par jour en cas d'infirmité, payer les soins du médecin et une pension annuelle de $365 \mathrm{~F}$ à l'âge de 55 ans.

En 1821, la société des Vrais Amis reçoit une subvention et quatre brevets d'encouragement donnés par Lebaron, directeur des sociétés philanthropiques de la Préfecture de la Seine. Une médaille pour la naissance du duc de Bordeaux, frappée par Louis XVIII, est déposée dans la caisse de la société11. Le caractère de simple prévoyance - et non de résistance - affiché par les Vrais Amis et leur soumission au régime politique en place expliquent sans aucun doute la facilité avec laquelle les bouchers ont obtenu du préfet de police l'autorisation pour leur société. Dans une circulaire du 31 octobre 1812 adressée aux préfets, le ministère de l'Intérieur prône une certaine tolérance pour les sociétés de prévoyance et de secours mutuels, alors que les coalitions ouvrières sont réprimées ${ }^{12}$. Bernard Gibaud explique très bien comment une telle création a été possible en 1820:

Matrot (1830-1913) a été administrateur et président d'honneur des Vrais Amis. A sa mort, il a fait don d'un immeuble (24 rue de Turbigo à Paris) aux Vrais Amis, " pour servir à la propagande de la Mutualité, et apporter un soutien aux vieux jours des sociétaires malheureux ».

${ }^{10}$ Hubbard Gustave-Nicolas, De l'organisation des sociétés de prévoyance ou de secours mutuel et des bases scientifiques sur lesquelles elles doivent être établies, Paris, Guillaumin, 1852, 243 p.

${ }^{11}$ Louis XVIII était le «Président perpétuel » de la Société philanthropique de Paris, la plus active des sociétés de secours sous la Restauration, qui bénéficie du patronage royal à partir de 1818. Gibaud Bernard, «La société philanthropique de Paris ou les paradoxes du patronage aristocratique », Revue de l'économie sociale, n¹3, 1988, pp. 177-183.

12 Soubiran-Paillet Francine, L'invention du syndicat (1791-1884) : itinéraire d'une catégorie juridique, Paris, LGDJ, 1999, p. 26. 
«La renaissance de la vie associative dans les métiers, au début du XIX siècle, en dépit du code pénal napoléonien et de son redoutable article 29113, répond à des besoins incompressibles de protection que l'Etat n'a nullement la volonté, ni les moyens de prendre en charge. Les sociétés de secours mutuels bénéficient d'une relative tolérance, à la condition de limiter leurs activités aux seules tâches d'assistance. La mise en place d'un barrage filtrant tend à les différencier en deux grandes catégories : les sociétés vouées exclusivement aux secours et les sociétés plurifonctionnelles de résistance et de prévoyance. Ces dernières, perçues encore récemment comme la forme générique de la mutualité en raison de leur rôle d'antichambre du syndicalisme ouvrier, ne représentent qu'une fraction minoritaire de la gestation mutualiste $»^{14}$.

Le soutien apporté en 1821 aux Vrais Amis par le directeur des sociétés philanthropiques de la Préfecture de la Seine n'est pas anodin. Dans les grandes villes comme Paris, la mutualité d'entraide est soutenue par les sociétés philanthropiques, dont les dirigeants sont souvent des aristocrates ${ }^{15}$. Ainsi, sous la Restauration, la Société philanthropique de Paris «favorise la création de plus de 150 groupements mutualistes professionnels et fait adopter le terme unificateur de société de secours mutuels ${ }^{16}$ ». Les Vrais Amis participent de cette dynamique. L'origine ouvrière de l'initiative de la création ne doit pas masquer le fait que les Vrais Amis ne sont absolument pas une société de résistance mais bien une société de prévoyance, nostalgique de la solidarité que l'on trouvait dans les anciennes confréries. Bernard Gibaud résume ainsi cette nostalgie pour l'Ancien Régime : «Les aristocrates monarchistes tendent à adopter une position sensiblement différente de la bourgeoisie économique sur le phénomène associatif. On ne souhaite, certes pas, encourager l'association, cheval de Troie de la coalition ouvrière, mais redonner vie à des conceptions hiérarchisées de l'organisation sociale, faisant une place relative aux liens solidaires ${ }^{17}$ ».

Si la création des Vrais Amis en 1820 répond à des préoccupations sociales et « humanitaires », ce sont plutôt des motivations politiques qui guident les patrons bouchers lorsqu'ils créent en 1824 une autre société de secours mutuels, «la Société de prévoyance et de secours mutuels des ouvriers et employés de la Boucherie de Paris ». Par commodité, on la nommera «la société du Syndicat». Depuis 1802-1811 en

${ }^{13}$ L'article 291 du code pénal napoléonien est ainsi rédigé: «Nulle association de plus de 20 personnes, dont le but sera de se réunir tous les jours ou à certains jours pour s'occuper d'objets religieux, littéraires, politiques ou autres, ne pourra se former qu'avec l'agrément du gouvernement et sous les conditions qu'il plaira à l'autorité publique d'imposer à la société ».

${ }^{14}$ Gibaud Bernard, Mutualité, assurances..., op. cit., p. 14.

15 Sur cet aspect, je renvoie à Duprat Catherine, Usage et pratiques de la philanthropie. Pawvreté, action sociale et lien social à Paris au cours du premier XIXe siècle, Paris, Comité d'Histoire de la Sécurité sociale, 1997, volume 1, pp. 327-403.

${ }^{16}$ Gibaud Bernard, Mutualité, assurances..., op. cit., p. 15.

17 Ibid. 
effet, les bouchers parisiens disposent d'une chambre syndicale, situation exceptionnelle au regard de la loi Le Chapelier. En 1802, Bonaparte a établi un système règlementaire strict pour le commerce du pain et de la viande à Paris, avec un Bureau et une Caisse de la Boucherie : les bouchers doivent obtenir une autorisation administrative pour s'installer (avec un cautionnement obligatoire), l'approvisionnement en bestiaux s'effectue sur des marchés obligatoires, $\operatorname{etc}^{18}$... La restauration des privilèges est parachevée en 1811 avec la limitation du nombre des étaux dans la capitale. A partir de 1811, tous les bouchers de Paris doivent recourir à la Caisse de Poissy pour leurs achats en bestiaux et la profession est organisée par un Syndicat qui gère les problèmes quotidiens. La création d'une société de secours mutuels s’inscrit donc pour les bouchers parisiens dans un contexte particulier, proche de la structure corporation - confrérie existant avant la Révolution. D’ailleurs, quand Louis Goyard décrit la genèse - plus ou moins légendaire de la société du Syndicat, il inscrit son récit dans la lutte de certains bouchers contre la règlementation contraignante néo-corporative mise en place depuis 1811. Cette proximité est très éclairante.

En 1821, quatre étaliers adressent une pétition à Louis XVIII pour demander la liberté pleine et entière du commerce de la boucherie. Une ordonnance d'octobre 1822 donne en partie satisfaction aux partisans de la liberté, en supprimant un article du décret du 13 juin 1808 qui obligeait les bouchers qui voulaient s'établir à acheter deux étaux et à en fermer un (dans la perspective de la diminution du nombre d'étaux dans Paris). En 1823, le syndicat de la Boucherie demande la remise en vigueur de cet article et cherche à nuire aux quatre pétitionnaires. «Pour toute réponse le préfet de police fit publier la pétition des quatre étaliers, qui était toujours restée ignorée et fut alors connue de toute la Boucherie de Paris. Ce fut un événement considérable, les avis étaient partagés en deux camps, les quatre étaliers furent mis un peu au ban de la corporation; mais à cette époque on manifestait déjà en banquetant $»^{19}$. Toujours en 1823, pendant un banquet, les étaliers réitèrent leur demande d'abolition du privilège et réclament à nouveau la liberté pleine et entière du commerce de la boucherie. Suite à ce banquet, le syndicat réagit en luttant contre les «factieux » et adresse une demande en juin 1823 au préfet de police pour créer la «Société de prévoyance et de secours mutuels des ouvriers et employés de la Boucherie de Paris ». Les patrons bouchers auraient donc créé cette mutuelle pour mettre fin aux revendications libérales d'un groupe de garçons bouchers. Pourquoi ces étaliers n'ont-ils pas tout simplement rejoint les Vrais Amis ? Sans doute à cause des tarifs trop élevés qui ne permettent pas à la plupart des employés d'y adhérer. Mais surtout, les motivations profondes de la création de la société du Syndicat sont différentes de celles qui ont mené à la fondation des Vrais Amis.

18 Pour plus de détails, je renvoie à mon article, «Les formes d'intervention des pouvoirs publics dans l'approvisionnement en bestiaux de Paris : la Caisse de Poissy de l'Ancien Régime au Second Empire ", Cahiers d'économie et de sociologie rurales, 2005, nº74, pp. 49-78.

${ }^{19}$ Matrot Henry, op. cit., p. 40. 
Les statuts de la mutuelle du syndicat sont déposés en décembre 1823. Le préfet de police donne sa permission le 3 février 1824. La première réunion de la société se tient en juin 1824. Le conseil d'administration compte deux membres du syndicat et deux marchands bouchers. Les activités de la mutuelle débutent le 1er juillet 1824. La première assemblée générale se tient le 28 septembre 1824, avec Taffoureau comme président et quatre adjoints choisis parmi les sociétaires (Touzeau, Dolbel, Caron, Bonnet). L'équipe dirigeante est quasiment identique à celle des Vrais Amis. Taffoureau est à la fois président de la société du Syndicat et trésorier des Vrais Amis. Caron est président des Vrais Amis et adjoint de la société du Syndicat. Bonnet est administrateur des Vrais Amis et adjoint de la société du Syndicat. Dolbel est adjoint de la société du Syndicat et devient trésorier des Vrais Amis en mars 1829, quand Taffoureau, malade, démissionne avant de mourir en 1829 à l'âge de 44 ans. Un autre signe de la proximité des deux mutuelles est évident: en avril 1825, la société du Syndicat adopte un nouveau règlement, copié sur celui des Vrais Amis. Si l'on veut intégrer les deux sociétés dans la typologie d'Henri Hatzfeld, qui distingue trois formes de mutualité au XIXe siècle (ouvrière, populaire et patronnée), il est clair que les deux organisations appartiennent à la catégorie de la «mutualité patronnée » ${ }^{20}$.

Comment fonctionne la société de secours mutuels du syndicat? Pendant les six premiers mois d'existence de la mutuelle, les candidats sont admis entre 18 et 45 ans. La limite d'âge est fixée ensuite à 30 ans. Le droit d'admission est de 10 francs (avec une pénalité pour les plus de 30 ans). La cotisation est de 75 centimes par semaine pour chaque sociétaire. Les prestations sont les suivantes: une indemnité journalière de 2,50 F pendant la maladie ou les blessures, une indemnité de 1,50 F par journée de convalescence, le paiement des soins du médecin et des médicaments, l'allocation d'une somme pour les funérailles, une pension de retraite de $250 \mathrm{~F}$ après 55 ans $^{21}$. Un secours exceptionnel est accordé en 1824 à douze vieux garçons bouchers infirmes et non sociétaires $(172,50 \mathrm{~F}$ accordés en quatre versements à chacun). Le 16 octobre 1824, plusieurs garçons bouchers de plus de 45 ans sont admis dans la société, mais les années de cotisation sont plus longues pour eux. Quand la société a été fondée en 1824, le syndicat de la Boucherie lui a versé $10000 \mathrm{~F}$. Le 8 janvier 1825, le syndicat s'engage à verser $2000 \mathrm{~F}$ de subvention annuelle à la mutuelle.

La principale différence entre les deux sociétés se trouve dans le mode de gestion. Les Vrais Amis ont une gestion financière beaucoup plus saine : le recrutement suit des normes strictes, les membres paient des cotisations plus élevées et ne peuvent compter sur aucune aide extérieure. Par contre, la société du Syndicat a une gestion déficitaire, se permet d'accepter des membres non solvables ou peu rentables, car elle compte sur le syndicat pour combler son déficit chronique. Les Vrais Amis sont viables économiquement alors que l'autre société ne survit que par les aides et subventions du syndicat. Quand le syndicat de la Boucherie est supprimé entre 1825 et 1829, il semble évident que ce fonctionnement est de

${ }^{20}$ Hatzfeld Henri, « Note sur la mutualité au XIX ${ }^{\mathrm{e}}$ siècle », Prévenir, n 9 , mai 1984.

${ }^{21}$ La pension de retraite passe à $365 \mathrm{~F}$ en 1833. 
facto remis en cause. Le préfet de police a une part de responsabilité car c'est lui qui autorise dans un rapport d'octobre 1824 le syndicat de la Boucherie à prélever $10000 \mathrm{~F}$ sur les intérêts des cautions pour les verser dans la caisse de la mutuelle ${ }^{22}$. Un texte de 1845 résume bien la situation : "La société est créée en 1824 par le Syndicat de la Boucherie de Paris, qui a versé dans la caisse de cette société, avec l'assentiment des électeurs du commerce, une somme de $10000 \mathrm{~F}$ pour former de suite un fonds convenable et faciliter l'admission des candidats qui avaient atteint l'âge de 40 ans lors de la formation de cette société $»^{23}$.

Quelle a été l'évolution des deux sociétés de secours mutuels sous la monarchie de Juillet? La société du Syndicat est toujours confrontée à de sérieuses difficultés financières, fort compréhensibles quand on sait que les conditions d'entrée y sont beaucoup plus souples que celles des Vrais Amis. Avant 1825, le Syndicat lui versait $2000 \mathrm{~F}$ chaque année. A cause de la suppression du Syndicat entre 1825 et 1829 et divers retards de paiements, la mutuelle est restée 9 ans sans rien toucher, soit une perte de 24000 F. Vers 1833, elle doit payer neuf pensions d'infirmité de 365 F. Aumont, syndic de la Boucherie, annonce que le Syndicat ne peut payer les 20000 francs réclamés : il verse $200 \mathrm{~F}$ par an pour chacun des membres arrivant à la pension et admis au-dessus de 40 ans (36 sociétaires). Vers 1835, le règlement de la mutuelle est modifié: la durée de cotisation passe à 25 ans pour être admis à la pension. Les discussions sur le tarif des cotisations vont bon train entre 1835 et 1844 . Bernard Gibaud rappelle que «la faible capacité d'épargne de la population laborieuse et l'absence d'un cadre juridique de référence pour la pratique mutualiste, exception faite de la loi du 22 juin 1835 autorisant des dépôts dans les caisses d'épargne, rendent précaire le fonctionnement des institutions d'entraide $»^{24}$.

Le 5 janvier 1836, trois cartes de dispensaire sont achetées pour procurer des médicaments gratuits aux sociétaires malades ${ }^{25}$. En 1836, plusieurs membres du Syndicat entrent dans la société. En 1839, la convention Aumont est révisée, car sur les 36 vieux sociétaires, 4 sont morts, 17 sont pensionnés et 15 seront bientôt pensionnés : la subvention de 200 F accordée par le Syndicat est réduite de moitié. Quand Purget devient syndic en 1841, il veut encore réduire les dépenses du Syndicat : la subvention annuelle est réduite de moitié pour les pensionnés. En 1842, pour suivre l'exemple des Vrais Amis, la société du

22 Rapport du préfet de police de Paris du 13 octobre 1824. Archives de la Préfecture de Police de Paris, DA 365, dossier n 1 sur la société de secours mutuels des ouvriers et employés de la Boucherie de Paris.

${ }^{23}$ Société de prévoyance et de secours mutuels de la Boucherie de Paris : règlement et tarifs, Paris, Lebègue, 1845. Archives de la Préfecture de Police de Paris, DB 401.

${ }^{24}$ Gibaud Bernard, Mutualité, assurances..., op. cit., p. 20.

${ }^{25}$ Sur « les services médicaux et pharmaceutiques » dispensés par les sociétés de secours mutuels au XIX ${ }^{\mathrm{e}}$ siècle et les débats sur la gratuité des soins et des médicaments, je renvoie aux éléments fournis par Jean Bennet, La mutualité à travers..., op. cit., pp. 129-145. 
Syndicat accorde aux veuves le droit de succéder à leur mari ${ }^{26}$. Une nouvelle réduction des subventions intervient en 1843: la situation financière de la mutuelle devient alors très fragile.

Dans le même temps, le rapprochement entre le Syndicat et la société des Vrais Amis devient de plus en plus évident: plusieurs syndics de la Boucherie, comme Aumont en 1834 et André Vesque en 1836, deviennent «membres doubles », c'est-à-dire qu'ils appartiennent aux deux mutuelles. Vers 1840, Caron père, président des Vrais Amis, obtient du Syndicat le droit de tenir les réunions dans la salle des séances du Syndicat, ce qui entraine une économie de locaux. Un signe est révélateur du rapprochement entre les deux mutuelles : vers 1836, Caron, président des Vrais Amis, est nommé président de la société du Syndicat, « contre son gré ». Etant inspecteur de la Boucherie (à l'abattoir du Roule), Caron est sous les ordres directs des délégués du commerce de la Boucherie: ces membres décident d'être absents aux assemblées générales de la société du Syndicat pour ne pas gêner son action. Par ailleurs, Goyard et Caron fils deviennent membres du Conseil d'administration de la société du Syndicat en décembre 1843. En 1844, quand Caron démissionne, son fils lui succède comme président de la société du Syndicat.

En 1844, la mutuelle du Syndicat se met à vendre des pensions : en versant $3829 \mathrm{~F}$ à 55 ans, la société verse une rente viagère de $365 \mathrm{~F}$ par an ${ }^{27}$. Cette somme de $3829 \mathrm{~F}$ peut sembler énorme, mais quand on sait que le boucher prenant sa retraite touche le remboursement des $3000 \mathrm{~F}$ de sa caution administrative, l'effort financier devient supportable. Le bilan comptable de la société demeure instable. En 1844, l'excédent de recettes n'est que de 1147 F. Le détail des recettes n'est pas lu dans les procèsverbaux de l'Assemblée Générale: le conseil d'administration accepte cette omission mais rechigne à cacher aux sociétaires la vraie situation financière de la société. En 1847, le déficit est de 576 F. En avril 1847, Caron fils démissionne de son poste de président et Goyard lui succède. La situation financière de la société du Syndicat est structurellement précaire sous la monarchie de Juillet; la Révolution de 1848 va lui apporter le coup de grâce.

${ }^{26}$ Jusqu'en 1852, les femmes sont rarement admises dans les sociétés de secours mutuels. Ainsi, à Paris, sur les 193 sociétés créées entre 1791 et 1830, 20 seulement acceptent les femmes (veuves la plupart du temps), ce qui donne un total de 127 femmes sur les 1996 mutualistes parisiens (6,3\%). Bennet Jean, $L a$ mutualité à travers..., op. cit., p. 191. Pour plus de détails, on peut consulter Dreyfus Michel, Les femmes et la Mutualité française de la Révolution française à nos jours, Paris, Editions Pascal, 2006, 126 p.

27 « La situation la plus critique pour la société du syndicat se produisit entre 1844 et 1849. Le syndicat avait cessé de remplir ses engagements et pour y remédier la société avait imaginé une sorte de "rachat global": à 55 ans on pouvait se constituer une rente viagère de 365 francs par an et par un versement de 3829 francs ». Bony Pierre, «Une doyenne...», op. cit., p. 15. 


\section{Les changements sous la Seconde République et le Second Empire}

Les deux sociétés, jumelles plus que rivales, vont évoluer dans un sens assez prévisible, celui du rapprochement. Les bases comptables sur lesquelles reposent les Vrais Amis étant beaucoup plus saines que celles de la société du Syndicat, c'est assez naturellement que la doyenne va absorber sa cadette. La remise en cause progressive du privilège des bouchers parisiens s'accélérant avec la mise en place de la Seconde République (réforme du droit d'octroi et mise en place d'une vente à la criée de la viande), le parrainage financier dont bénéficiait la société du Syndicat disparait, poussant celle-ci à fusionner avec les Vrais Amis.

La Révolution de 1848 sonne l'hallali de la société du Syndicat. Face aux différents problèmes qui frappent le Syndicat, le syndic refuse de payer la subvention de 1848. Le déficit de la société augmente d'autant plus. Goyard propose alors à Grosset, président des Vrais Amis depuis mars 1849, de réunir les deux mutuelles, mais la dette doit être apurée par le Syndicat. La société du Syndicat se livre à des manœuvres comptables pour truquer le bilan, en faisant entrer un grand nombre de cotisations arriérées. En 1849, le déficit s'élève à $1850 \mathrm{~F}$ : la liquidation semble proche, même si 50 nouveaux sociétaires entrent alors dans la société du Syndicat.

En 1849, le syndic de la Boucherie refuse tout d'abord d'apurer la dette de la mutuelle, car «les ressources du syndicat sont les intérêts des cautionnements, mais la situation est difficile ». Finalement, en 1850, le syndicat rembourse le déficit et accepte de payer les rentes des vieux pensionnés. La réunion des deux sociétés peut donc se conclure. Elle n'est pas difficile à mettre en place, tant les statuts, l'administration et le personnel sont quasiment identiques dans les deux mutuelles. Le projet est discuté dans chaque assemblée générale en avril 1851. La société du Syndicat se réunit en assemblée générale extraordinaire en mai 1851 et vote à l'unanimité pour la fusion. Les Vrais Amis se réunissent à leur tour en juin et acceptent la fusion à l'unanimité moins 5 voix. La réunion des deux sociétés est effective le 1er juillet 1851. Dans un article de la Revue municipale de 1849, Rilliot dresse un bilan comparatif des deux mutuelles.

Tableau : Bilan financier des deux sociétés de secours mutuels des bouchers en 1848

\begin{tabular}{|l|r|r|}
\cline { 2 - 3 } \multicolumn{1}{c|}{} & Vrais Amis & Société du Syndicat \\
\hline Somme en caisse au 1er janvier 1848 & $202337 \mathrm{~F}$ & $148107 \mathrm{~F}$ \\
\hline Montant reçu en 1848 (cotisations, amendes) & $12091 \mathrm{~F}$ & $11783 \mathrm{~F}$ \\
\hline Intérêt du capital & $9000 \mathrm{~F}$ & $6725 \mathrm{~F}$ \\
\hline Total & $223428 \mathrm{~F}$ & $166615 \mathrm{~F}$ \\
\hline Dépenses & $-11904 \mathrm{~F}$ & $-16331 \mathrm{~F}$ \\
\hline Capital au 31 décembre 1848 & $211524 \mathrm{~F}$ & $150284 \mathrm{~F}$ \\
\hline
\end{tabular}




\begin{tabular}{|c|c|c|}
\hline Composition du capital & Vrais Amis & Société du Syndicat \\
\hline Inscriptions de rentes $5 \%$ au capital de & $205256 F$ & $148348 \mathrm{~F}$ \\
\hline entre les mains du caissier & $2373 \mathrm{~F}$ & $1200 F$ \\
\hline entre les mains du secrétaire & $3895 \mathrm{~F}$ & $736 \mathrm{~F}$ \\
\hline Total & $211524 \mathrm{~F}$ & $150284 \mathrm{~F}$ \\
\hline
\end{tabular}

La situation financière de 1848 montre clairement que la société la plus ancienne, celle des Vrais Amis, est aussi la plus riche. Rilliot reconnaît que les deux sociétés ont presque le même nombre de membres (160 environ), mais « les Vrais Amis ont 9.000 livres de rente alors que l'autre n'en a que 6725 ». Les Vrais Amis n'ont que 39 pensionnaires, auxquels elle verse $9842 \mathrm{~F}$, alors que la société du Syndicat compte 54 pensionnaires, auxquels elle donne $14535 \mathrm{~F}$. Rilliot évoque la principale faiblesse de cette dernière : lorsqu'elle a été formée, « un certain nombre de membres ont été admis sans tenir aucun compte de leur âge pour fixer leur cotisation. La dotation faite par le Bureau de la boucherie n'a pas été assez forte pour compenser le déficit. Mais les deux sociétés sont prospères ». Ce dernier avis me semble peu fondé. Rilliot termine son article par quelques considérations morales, assurant que les statuts des deux sociétés sont «justes et moraux, établis sur des bases très solides ». Les bouchers sont de très bons travailleurs, presque jamais malades et ils ne vivent pas très vieux, "circonstances particulières qui allègent singulièrement les charges des sociétés, et qu'on doit attribuer sans doute à la force de leur alimentation $»^{28}$.

Quant à Louis Goyard, il dresse un bilan comparatif des deux sociétés au moment de leur fusion. En 1851, les Vrais Amis rassemblent 178 sociétaires pour un capital de 225.108 F. La société de secours mutuels du Syndicat compte 247 sociétaires, pour un capital de $149028 \mathrm{~F}$, auquel il faut ajouter $116190 \mathrm{~F}$ (aides du syndicat, économie des frais de gestion, retards de cotisation). Le capital réel s'élèverait donc à $265218 \mathrm{~F}$. Une fois la fusion accomplie, le nouvel ensemble compte 401 membres au $1^{\text {er }}$ janvier $1852^{29}$.

La nouvelle dénomination adoptée après la fusion est « Société de prévoyance et de secours mutuels de la Boucherie de Paris, dite des Vrais Amis ». Par commodité, nous l'appellerons «les Vrais Amis». L'assemblée générale unifiée du 8 juillet 1851 nomme le nouveau conseil d'administration : Grosset est président, Goyard trésorier, Heomet administrateur, et l'on compte sept adjoints. Il est décidé que chaque année, le mercredi de la Semaine Sainte, sera organisée une messe à Saint-Eustache pour le repos de l'âme

${ }^{28}$ Rilliot, « Société de secours mutuels de la boucherie de Paris », La revue municipale, 1849, p. 234-235.

${ }^{29}$ Sur le total des 425 membres des deux sociétés, il faut ôter 36 pensionnaires qui n’ont pas versé leur cotisation. Sur les 389 cotisants, il y a le cas particulier de 90 sociétaires «membres doubles » (abonnés aux deux sociétés). 
des sociétaires défunts et la prospérité de la société, avec un banquet au soir. L'existence d'une telle cérémonie religieuse montre bien l'attachement des bouchers aux valeurs catholiques ${ }^{30}$.

Il est paradoxal de constater que la société du Syndicat a souffert de la révolution de 1848, alors que la proclamation de la Seconde République a inauguré une période heureuse pour les sociétés de secours mutuels. Il est vrai que "l'activité des sociétés de secours mutuels bénéficie pendant deux ans de l'abrogation implicite de l'article 291 du code pénal et des dispositions aggravées de la loi de $1834^{31} »$. Mais, en février 1849, quand le débat sur les caisses de secours mutuels et de prévoyance est ouvert à l'Assemblée constituante par René Waldeck-Rousseau et Pierre Rouveure, les idées de couverture globale et de financement public obligatoire sont rejetées. Bernard Gibaud évoque une intervention « insolite » de l'Etat dans la protection sociale : la loi du 18 juin 1850 institue une Caisse nationale pour la retraite (avec des livrets individuels gérés par capitalisation) ${ }^{32}$. Enfin, la loi du 8 mars 1850 permet aux sociétés de secours mutuels de se faire déclarer d'utilité publique avec des avantages fiscaux, moyennant une tutelle administrative. Ce statut ne tente que 9 sociétés sur environ 200 existantes à Paris. Les Vrais Amis refusent d'appliquer cette disposition car ils veulent rester « autogérés ».

Quelles sont les conséquences de la mise en place du Second Empire sur l'évolution des Vrais Amis? La société reçoit 163 nouveaux membres en 1852. En mars 1852, le gouvernement réduit le rapport des rentes de 5\% à 4,12\%. Comme la société possède $18000 \mathrm{~F}$ de rentes, la perte de capital s'élève à $38160 \mathrm{~F}$. Cette perte est inattendue et douloureuse pour les bouchers car l'almanach de la Boucherie affirmait en 1848 que le placement en rente sur l'Etat «offre une garantie suffisante de leur prospérité́3 $^{3}$ ». Le 31 décembre 1855, le capital est augmenté de 100000 F. A partir de mars 1857, le paiement des pensions n'a lieu qu'après recouvrement des cotisations. Entre 1851 et 1861, la mutuelle réduit de moitié la réversibilité de la pension à la veuve, mais continue de verser, sur les encours, une pension entière jusqu'à la mort des veuves.

Le décret organique du 26 mars 1852 est très important car la mutualité est institutionnalisée. Ce décret « recentre la mutualité sur sa vocation d'entraide. Il lui apporte toute sorte d'encouragements. Il

\footnotetext{
${ }^{30}$ Sur ce point, je renvoie à mon article, «L'Eglise et les artisans : l’attachement des bouchers parisiens au catholicisme du 15e au 20e siècle », Revue d'Histoire Ecclésiastique, juin 2004, vol 99, pp. 371-390.

${ }^{31}$ La loi du 10 avril 1834 aggrave les sanctions contre les associations non autorisées. « Entre 1825 et 1848, 4460 membres de sociétés sont condamnés à des peines de prison pour délit de coalition, sans compter les affrontements sanglants liés au mutuellisme ». Gibaud Bernard, Mutualité, assurances..., op. cit., p. 20.

${ }^{32}$ Ibid., p. 27.

33 Almanach du commerce de la Boucherie, 1848, p. 5. BNF, V 27641.
} 
l'organise sur une base territoriale, et non plus par profession ou par classe sociale» ${ }^{34}$. Le cadre communal est préconisé car le cadre professionnel suscite la méfiance des pouvoirs publics. Le décret distingue trois types de sociétés de secours mutuels: les sociétés "approuvées », qui acceptent un président nommé par l'Empereur en échange de subventions ; les sociétés « autorisées », libres de choisir leur président mais non subventionnées et étroitement surveillées; et les sociétés reconnues d'utilité publique (définies dans le cadre de la loi du 8 mars 1850). Si l'on se fie aux propos de Louis Goyard, les Vrais Amis sont une société «autorisée » entre 1852 et 1857, «approuvée » ensuite. Les Vrais Amis demandent l'approbation du gouvernement en octobre 1857 et un décret impérial du 9 décembre 1857 nomme Grosset président de la société. Ce choix de l'approbation n'est pas fréquent à l'époque à Paris, car, comme le dit Patricia Toucas, «l'approbation se heurte à la résistance d'une mutualité traditionnellement corporative. En 1859, sur 383 sociétés dans le département de la Seine, 123 seulement sont approuvées »35. Comme l'explique Michel Dreyfus, les sociétés approuvées «bénéficient de privilèges fiscaux et de l'autorisation de placer aux Caisses d'épargne ainsi qu'à la Caisse des dépôts et consignations, les capitaux destinés à la constitution des pensions de vieillesse. Par l'intermédiaire de la Caisse des dépôts, l'Etat encourage, tout en la contrôlant, l'utilisation des fonds déposés par les mutualistes $\aleph^{36}$. En 1870, plus de 70\% des sociétés de secours mutuels françaises sont des sociétés approuvées ${ }^{37}$.

En 1858, Napoléon III décide de supprimer la Caisse de Poissy et le Syndicat de la Boucherie de Paris pour libéraliser le commerce de la viande dans la capitale : le système néo-corporatif mis en place en 1802-1811 est aboli. Cette décision est lourde de conséquences pour les Vrais Amis. Comme pendant les autres crises $(1825-1829,1848)$, le Syndicat refuse de payer les subventions du $1^{\text {er }}$ trimestre 1858. Le Conseil d'Etat soutient les réclamations des Vrais Amis. Suite à une transaction à l'amiable passée le 27 novembre 1860, les mandataires de la Boucherie versent 12000 F dans la caisse des Vrais Amis. En 18581860, les pertes de la société se situent entre $26667207 \mathrm{~F}$ et $36364837 \mathrm{~F}$. Suite à la suppression du Syndicat, les Vrais Amis doivent quitter les locaux de la Halle aux veaux et installent leur siège au 8 rue Larrey. Les séances sont tenues dans la salle Saint-Jean de l'Hôtel de ville, mise à disposition par le préfet Haussmann. La mutuelle se trouve donc dans une situation matérielle délicate à partir de 1858 .

On trouve dans les archives de la préfecture de police de Paris un dossier sur la « liquidation de la caisse des retraites des bouchers et employés du Syndicat (1858-1860) $»^{38}$. Il s'agit de la vente d'une

\footnotetext{
${ }^{34}$ Fridenson Patrick, «Le conflit social», in Burguière André et Revel Jacques (dir.), Histoire de la France: l'Etat et les conflits, tome 3 : les conflits, Paris, Seuil, 1990, p. 402.

35 Toucas Patricia, «Les sociétés approuvées du Second Empire », in Dreyfus Michel, Gibaud Bernard et Gueslin André (dir.), Démocratie, solidarité et mutualité : «autour de la loi de 1898 », Economica, 1999, p. 20.

${ }^{36}$ Dreyfus Michel, Liberté, égalité, mutualité..., op. cit., p. 41.

${ }^{37}$ Ibid., p. 43.

38 Archives de la Préfecture de police de Paris, DA 365, dossier 4.
} 
inscription de rente aux Vrais Amis, dont le capital est formé par des retenues sur les traitements des employés de la Boucherie (retenue de 5\%). Ce projet de caisse de pension avait été approuvé par les électeurs de la Boucherie en décembre 1834, mais avait été rejeté par la préfecture de police en 1835 et 183639. En 1858, 29 personnes (dont 16 veuves) sont concernées par ces pensions qui se trouvent liquidées entre 1858 et 1860 .

Dans son enquête de 1860 sur la situation de l'industrie parisienne, la Chambre de commerce de Paris indique que presque tous les ouvriers de la boucherie «font partie d'une société de secours mutuels, qui, moyennant une cotisation mensuelle, leur assure des ressources en cas de maladie et une retraite à l'âge de 55 ans $»^{40}$. Ce tableau est idyllique mais erroné. Sachant que Paris compte 1132 bouchers et 2600 ouvriers en 1860, cela signifierait que les Vrais Amis rassemblent 3732 adhérents! A moins qu’il ne s'agisse de la «caisse des retraites des bouchers et employés du Syndicat », dissoute en 1858 et en voie de liquidation en 1860 ? Nous ne connaissons pas la proportion des bouchers qui cotisent aux Vrais Amis, mais nous savons que les effectifs de la mutuelle n'ont jamais du dépasser 500 personnes au XIX ${ }^{\mathrm{e}}$ siècle. Les Vrais Amis regroupent 400 adhérents en 1852, 450 en 1874, 427 en 1880 et 376 en 188841.

Les Vrais Amis sont reconnus d’utilité publique par un décret du 22 décembre 1866. En 1868 se constitue une Chambre syndicale patronale de la Boucherie de Paris, tolérée par le préfet de police (alors que les garçons bouchers doivent attendre 1886 pour en disposer). Ce syndicat " rénové » pourra ponctuellement apporter une aide financière aux Vrais Amis mais en aucun cas il n'apportera une subvention régulière, comme c'était le cas jusqu'en 1858. Cela signifie qu'à partir de 1858, les Vrais Amis sont soumis aux mêmes exigences comptables que n'importe quelle autre mutuelle. La situation est « normalisée » à partir du Second Empire.

\section{La société des Vrais Amis sous la Troisième République}

En 1871, des garçons bouchers parisiens vont tenter de créer leur propre société de secours mutuels, dans une logique d'organisation ouvrière contre les intérêts patronaux. Le contexte social est lourd car Thiers vient d'écraser la Commune de Paris pendant la Semaine sanglante (21-28 mai 1871). Au cours de l'été 1871, la «Chambre syndicale des ouvriers et employés de la Boucherie » projette de créer la

39 Un arrêté du préfet de police du $1^{\text {er }}$ avril 1848 décide de former un fonds destiné aux pensions de retraite pour les garçons bouchers de Paris. C’est sans doute cette caisse de retraite qui est liquidée en 1858-1860.

${ }^{40}$ Chambre de commerce de Paris, Statistique de l'industrie à Paris résultant de l'enquête faite par la Chambre de commerce pour l'année 1860, Paris, 1864, p. 14.

41 Société de prévoyance et de secours mutuels de la Boucherie de Paris (les Vrais Amis), Paris, Wattier, 1889, p. 10. Archives de la Chambre de Commerce de Paris, 352126. 
Fraternelle, une société de secours mutuels très éphémère (qui disparait en 1872), mais qui suscite de vives inquiétudes chez les patrons bouchers. En juin 1872, la société compte 70 membres et propose de créer un bureau de placement gratuit pour les garçons bouchers. Le dossier sur la Fraternelle présent à la préfecture de police est assez pauvre. Quelques coupures de presse nous renseignent un peu plus ${ }^{42}$.

Dans La Vérité du 31 août 1871, le vice-président de la Fraternelle, Héroult, signe un article de défense des buts de la société face aux attaques patronales. La Fraternelle se présente comme une œuvre de solidarité, «afin de nous entraider et nous secourir, en cas de blessures ou de maladie, et de remplacer pour beaucoup de jeunes gens la famille éloignée; ensuite d'affranchir tous nos sociétaires de l'exploitation des bureaux de placement». A mots couverts, on reconnait dans ces propos le principe d'une société ouvrière de résistance qui lutte contre les abus des placeurs privés. La méfiance patronale semble alors tout à fait justifiée. Pourtant, toute idée de lutte de classe (ou de recours à la grève) est clairement écartée par Héroult: «Loin d'être en désaccord avec les intérêts de messieurs les patrons, attendu que la sympathie et l'union sont nécessaires à nos intérêts communs, puisque la prospérité de l'un est le résultat du travail et de la coopération de l'employé et par cela même augmente son bien-être. Il est donc clairement illogique que les membres de la Société combattent ou entravent, par quelques moyens que ce soit, le commerce, le travail ou l'avenir des maitres de maison. Voilà pourquoi nous avons tenu à honneur de détruire une imputation, qui peut être très préjudiciable à la prospérité de notre Société ».

De même, Héroult récuse tout projet de coopérative ouvrière. «On a répandu le bruit que les membres de la Société s'étaient réunis dans le but d'ouvrir des boucheries sous le titre d'Association fraternelle des employés de la Boucherie, et de faire une concurrence efficace aux anciennes maisons. Nous, membres de la Société, protestons de la manière la plus formelle contre cette exploitation du titre de notre Société, tandis que celle-ci y est restée complètement étrangère ; il est vrai que quelques-uns d'entre eux sont avec nous, mais ils ont agi en cela sans la participation et contre l'assentiment du reste des membres de la dite Société ; d'ailleurs, des reproches énergiques leur ont été adressés à l'Assemblée générale qui a eu lieu, salle de la Redoute, le $1^{\text {er }}$ août, à la suite de laquelle nous avons pris la résolution de protester par la présente et par tous les autres moyens en notre pouvoir, afin de mettre la Société et ses membres à l'abri d'une responsabilité qui semble vouloir retomber sur elle; et pour assurer toute la boucherie parisienne, que tous nos efforts ne tendront qu'à resserrer les bons rapports qui doivent unir la corporation tout entière, et à travailler de toutes nos forces à mériter l'estime et la considération de ceux dont nous sommes et voulons rester les fidèles et dévoués serviteurs ». Le ton de l'article se révèle assez curieux! Mais si le bureau de placement de la Fraternelle veut avoir quelques chances de réussite, la confiance des patrons bouchers est incontournable, sinon la tentative est vouée à l'échec.

En avril 1872, le conseil d'administration de la Fraternelle propose aux membres de la mutuelle « de constituer une Chambre syndicale des garçons bouchers », qui « aurait pour objet principal le placement

\footnotetext{
${ }^{42}$ Les articles de presse utilisés se trouvent dans le dossier sur la Fraternelle. Archives de la Préfecture de police de Paris, BA 1409.
} 
des garçons bouchers, qui pourrait s'effectuer dans des conditions de prix très modiques. Il a été calculé qu'il suffirait d'un versement mensuel de 50 centimes par chaque membre adhérent à la chambre syndicale pour couvrir les frais d'administration $»^{43}$. Le projet fait long feu.

Les membres de la Fraternelle tentent donc en 1871-1872 d'organiser rationnellement les différentes revendications ouvrières du moment : disposer d'une caisse d'assurance maladie et d'un bureau de placement gratuit. Ce dernier point sera d'ailleurs central dans la constitution d'un syndicalisme ouvrier autonome dans les années 1880. Entre 1890 et 1914, les syndicalistes ouvriers dénonceront assez souvent les Vrais Amis comme "société écran" permettant aux patrons bouchers de disposer d'un bureau de placement «clandestin »" Ces éléments permettent d'affirmer que l'identité paternaliste des Vrais Amis, présente dès sa fondation, demeure très forte à la fin du XIXe siècle.

L'éphémère existence de la Fraternelle n'a sans doute pas eu beaucoup d'impact sur l'équilibre financier des Vrais Amis, mais par contre la guerre de 1870 les a fragilisés. Pendant la lutte contre la Prusse, 204 jeunes membres de la société sont appelés sous les drapeaux : deux trouvent la mort et quatre sont blessés. Après la fin de la guerre, des dons permettent de renflouer la caisse de la mutuelle. En 1871, Alphonse Bernard Greyveldinger, ancien marchand boucher, lègue $3000 \mathrm{~F}$ à la société.

Louis Goyard indique que, pour prospérer, il faut que le revenu annuel couvre la moitié du service des pensions, et que la société doit augmenter le nombre de ses membres de $10 \%$ par an, à cause de la perte de $8 \%$ (démissions, radiations, décès). Hors, la société n'augmente que de $2 \%$ : elle doit recevoir 55

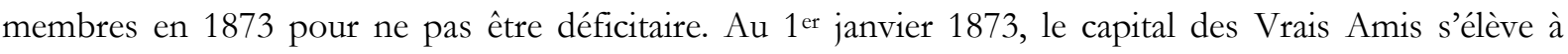
$539120 \mathrm{~F}$, dont $27215 \mathrm{~F}$ de rentes. En janvier 1875, l’Assemblée générale des Vrais Amis vote à l'unanimité la création d'une caisse de réserve. Dans une liste des membres honoraires fondateurs de cette caisse, dressée en 1889, on trouve diverses professions : des bouchers de détail et en gros, un facteur à la criée en viande, un facteur aux abats, des restaurateurs. On y remarque également des professionnels qui

43 Article du 20 avril 1872 sur la Fraternelle. Archives de la Préfecture de police de Paris, BA 1409.

${ }^{44}$ Par exemple, en novembre 1900, Bertrand Torny dénonce les agissements des Vrais Amis, qui « brave la loi en faisant apposer dans Paris et la province, des affiches invitant les parents nécessiteux à placer leurs enfants dans le dur métier qui est celui d'apprenti boucher. Ces affiches rédigées en termes mielleux leur dore la pilule par ces belles promesses telles que: Gagnant de suite, nourri et couché (l’on sait comment). Si toutes ces belles promesses étaient tenues, nous n'aurions pas à nous plaindre de cet état de choses ; malheureusement, il n'en est rien; cette manœuvre est digne de gens inhumains qui n'aspirent qu'aux honneurs sans se préoccuper des conséquences qui en découlent. Ils apportent volontairement de nouvelles victimes au chômage, dans l'espoir de trouver un personnel plus souple aux exigences patronales, car la misère est souvent escomptée par les mauvais patrons ». La Boucherie ouvrière, novembre 1900. 
traitent avec les bouchers, comme un bandagiste, un épurateur d'huiles, un imprimeur ou un serrurier qui s'occupe de la vente de fonds et de placement du personnel. Les soutiens apportés à la société de secours mutuels semblent donc assez variés. On peut aisément imaginer que la générosité des donateurs est récompensée par des relations privilégiées avec les adhérents de la mutuelle et du syndicat, et sans doute de la réclame gratuite dans les publications professionnelles. C'est probablement pour garder sa clientèle que H. Brunon, commissionnaire en cuirs, a fait un don de $2000 \mathrm{~F}$ aux Vrais Amis. Les membres libres et membres honoraires renoncent à l'indemnité de maladie ou d'infirmité, mais conservent le droit à la pension de retraite. Ces membres ont un « but humanitaire » : ils donnent l'exemple de la prévoyance et de la solidarité aux employés de la Boucherie.

La mutuelle conserve des liens étroits avec le syndicat patronal de la Boucherie (fondé en 1868). Garde, directeur du Fondoir central, et Douillet, juge suppléant au tribunal de commerce et ancien président du Syndicat des bouchers, sont des membres honoraires de la société. Au milieu des années 1880, la Chambre syndicale de la Boucherie de Paris, présidée par Lioré, fait un don de $800 \mathrm{~F}$ aux Vrais Amis. Dans les années 1880, le Bal de la Boucherie est organisé au profit de la caisse de réserve de la mutuelle. En juillet 1881, la société des Vrais Amis verse $100000 \mathrm{~F}$ à son fonds de retraite. Quand un concert lyrique est organisé en décembre 1887 au profit de la caisse de réserve des Vrais Amis, il a lieu dans la salle des conférences du n ${ }^{\circ} 10$ de la rue de Lancry, siège de l’Union générale du Commerce et de l'Industrie, fondée en 1858 et ancêtre de la Confédération nationale du patronat français. Dans les années 1890 et jusqu'en 1914, le Journal de la Boucherie de Paris n’omet jamais d'annoncer les assemblées générales et la fête annuelle des Vrais Amis" ${ }^{45}$. Ainsi, le 27 février 1896, le bal de la Boucherie, grande fête de bienfaisance au profit des Vrais Amis, se tient à l'Hôtel continental ${ }^{46}$.

La société modifie ses statuts en 1877 et en 1883. En 1879, les Vrais Amis obtiennent un diplôme d'honneur pour leur participation à l'exposition de 1878 : ils sont classés au huitième rang sur les 5923 sociétés de secours mutuels existantes en France. Les récompenses officielles s'accumulent sur les dirigeants de la mutuelle ${ }^{47}$. Lors de la cérémonie de 1878 où Alfred Hersant et Paul Matrat reçoivent leur médaille, le vice-président de la mutuelle, Matrot, improvise un discours aux côtés de Leroy-Daniel,

${ }^{45}$ Sur l'évolution du rôle des banquets et fêtes organisés par les mutuelles sous le Second Empire et la Troisième République, nous renvoyons à Michel Dreyfus, «La fête en mutualité », in Corbin Alain, Gérôme Noëlle et Tartakowsky Danielle (dir.), Les usages politiques des fêtes aux XIXe-XXe siècles, Paris, Publications de la Sorbonne, 1994, pp. 252-257.

46 Journal de la Boucherie de Paris, février 1896. BNF, Jo A 328.

47 Sous le Second Empire, des médailles récompensent les «généreux bienfaiteurs » des mutuelles. L'attribution des médailles (or, argent, vermeil, etc.) se fait «selon un système compliqué et minutieux qui ne commencera à se limiter qu'après la Seconde Guerre mondiale sans pour autant disparaitre complètement ». Dreyfus Michel, « La fête en mutualité », op. cit., p. 254. 
président de la Chambre syndicale de la Boucherie de Paris. Cette remise de médaille se tient à la salle de la Redoute, annexe de la Bourse du Travail, ancien siège de la franc-maçonnerie.

L'approbation administrative des Vrais Amis est renouvelée en décembre 1884. Le siège de la société se trouve alors au 2 rue de la poterie (Paris Ier), siège du syndicat patronal des bouchers. On peut devenir participant actif dès 16 ans : la cotisation augmente avec l'âge. Les objectifs de la mutuelle sont réaffirmés en 1884 : procurer en cas de maladie les soins d'un médecin et les médicaments nécessaires; payer une indemnité journalière pendant les maladies et blessures $(2,50 \mathrm{~F}$ par jour de maladie et 1,50 F par journée de convalescence); servir une pension de retraite, réversible pour partie sur la tête de la veuve ; assurer des funérailles convenables ${ }^{48}$.

Les Vrais Amis fondent un bulletin mensuel en janvier 1887 mais sa publication cesse en décembre 1888 à cause du " peu d'empressement des sociétaires à souscrire à l'abonnement de cette feuille » (recette annuelle de $400 \mathrm{~F}$ pour une dépense de $700 \mathrm{~F}$ ). Des protestations s'expriment au conseil d'administration contre le choix des insertions payantes parues dans le journal de la Chambre syndicale de la Boucherie. Les responsables de la mutuelle rappellent que la publicité est nécessaire pour obtenir de nouvelles adhésions. L'arrêt de la publication du bulletin mensuel en décembre 1888 et la mort de Louis Goyard en mars 1888 marquent la fin d'une source documentaire importante sur les Vrais Amis. C'est effectivement dans le bulletin mensuel que Goyard a publié par morceau son ouvrage sur l'Origine et le développement des sociétés de secours mutuels, qui est tout simplement une chronique des Vrais Amis entre 1820 et 1873 et la principale source d'information de cet article. Nous connaissons beaucoup moins bien l'évolution des Vrais Amis après 1889. En 1902, la société de secours mutuel de la Boucherie de Paris se dote à nouveau d'un organe de presse, La Mutualité corporative, qui aurait été «le plus fort tirage des journaux corporatifs» de la mutualité française, avant de disparaître en 191449.

Outre la tentative avortée de création de la Fraternelle en 1871-1872, les Vrais Amis voient naitre diverses sociétés concurrentes après 1870. Le 1er août 1878 sont déposés les statuts d'une société de secours mutuels «libre», la Caisse de secours des ouvriers de la maison Bernard, qui regroupe 66 personnes en 1905 et est présidée par le boucher Bernard ${ }^{50}$. Les patrons charcutiers créent avec succès leur mutuelle en 1879, l’Union de la Charcuterie, qui traverse tout le XXe siècle ${ }^{51}$. Une société éphémère a

${ }^{48}$ Dossier sur la mutuelle des Vrais Amis (1884). Archives de la Préfecture de police de Paris, BA 1409.

${ }^{49}$ Bennet Jean, La mutualité à travers..., op. cit., p. 175.

50 Archives de la Préfecture de police de Paris, DB 246.

${ }^{51}$ L’Union de la Charcuterie est fondée le 28 novembre 1879 par M. Bouchard. Entre 1936 et 1986, la mutuelle des charcutiers est dirigée par A. Guiot, qui organise la médecine du travail des charcutiers après 1945 avec la médecine préventive Bachaumont. La mutuelle élargit son champ d'action en 1967 et devient l'Union de la Charcuterie et de l'Alimentation française. Elle élargit encore sa clientèle en 1974 en 
existé : la «Solidarité de la Boucherie », société mutualiste autorisée en mars 1882 et rayée en mars 1888, sur laquelle nous ne savons rien ${ }^{52}$. Le ${ }^{\text {er }}$ septembre 1892 est fondée la société de secours mutuels des ouvriers de la Boucherie en gros, qui siège à la Villette (176 rue de Flandre). Le 16 août 1897 est fondée la société de secours mutuels des ouvriers boyaudiers, siégeant également à la Villette (176 rue de Flandre).

L'enquête de l'Office du travail de 1894 sur les associations professionnelles ouvrières indique que le secteur de l'alimentation à Paris compte 147 syndicats (17 474 membres), 75 sociétés de secours mutuels (10 263 membres) et deux coopératives (12 sociétaires) $)^{53}$. En France, les bouchers disposent de huit sociétés de secours mutuels, qui rassemblent 978 membres. Si l'on considère que les Vrais Amis doivent compter entre 500 et 600 adhérents en 1894, cela signifie que les autres mutuelles sont très confidentielles. On peut en conclure que la mutuelle des Vrais Amis constitue une belle réussite, exceptionnelle au niveau national.

Une enquête de l'Office du Travail de 1893 indique l'existence d'une mutuelle originale à la Villette : « La tentative la plus curieuse, parallèle au syndicat est la « Mutualité de la boucherie en gros » dirigée par un conseil d'administration à part. Cette caisse assure les accidents, non seulement en cas de responsabilité patronale, vis-à-vis des tiers, en cas de la faute du patron, mais aussi en cas de risques professionnels. L'ouvrier blessé pendant son travail a droit aux soins gratuits (médecin et médicaments) et à une indemnité de 2 francs par jour. Si sa faute seule a amené l'accident, le même secours peut lui être alloué par bienfaisance. Les patrons payent seuls la cotisation. Une tentative de retenue mensuelle sur le salaire des ouvriers n'a pas réussi. Avant la fondation de cette caisse, les chevillards s'adressaient (les deux tiers environ n’ont pas abandonné leur vieille habitude) à la Caisse des familles, 4 rue de la Paix ॥4 $^{54}$

Nous avons donc suivi le destin des Vrais Amis entre 1820 et 1898. Le cas de cette société de secours mutuels ne peut sans doute pas être généralisé à l'ensemble des artisans français car les bouchers parisiens ont bénéficié d'un statut professionnel exceptionnel entre 1802 et 1858 (avec l'établissement d'un système néo-corporatif qui a permis d'importantes aides financières) puis du puissant soutien de la Chambre syndicale patronale de la Boucherie de Paris, en activité dès 1868. La forte structuration du métier de boucher à Paris est sans aucun doute la principale raison qui explique le succès et la pérennité de la société des Vrais Amis au XIXe siècle, alors que tant d'autres tentatives similaires ont échoué. La création en 1930 de la Caisse primaire d'assurances sociales de la boucherie de Paris, puis les grandes

devenant l'UMIAD (Union Mutualiste Interprofessionnelle de l'Alimentation et Dérivés) puis en 1989 en devenant la MPI (Mutuelle Prévoyance Interprofessionnelle).

52 Archives de Paris, 1315 W 112.

${ }^{53}$ Ministère du commerce, Office du Travail, Les associations professionnelles owvrières, tome I : Alimentation, Paris, 1899, p. 276.

${ }^{54}$ Office du Travail, La petite industrie (salaires et durée du travail), tome 1: l'alimentation à Paris, Paris, Imprimerie nationale, 1893, p. 210. 
réformes sociales de l'après 1945 (notamment la mise en place en 1948 de la CARBOF, caisse obligatoire de retraite et de prévoyance de la boucherie) vont profondément modifier le fonctionnement et la raison d'être des Vrais Amis, qui deviennent une simple mutuelle complémentaire de retraite facultative. La baisse progressive du nombre des adhérents, avec notamment le passage fatidique sous le seuil des 5000 cotisants, entraîne en 1988 la mise en liquidation de la caisse autonome par répartition des Vrais Amis.

Nota : Une photo de la bannière des Vrais Amis en 1889 peut servir à illustrer cet article. 


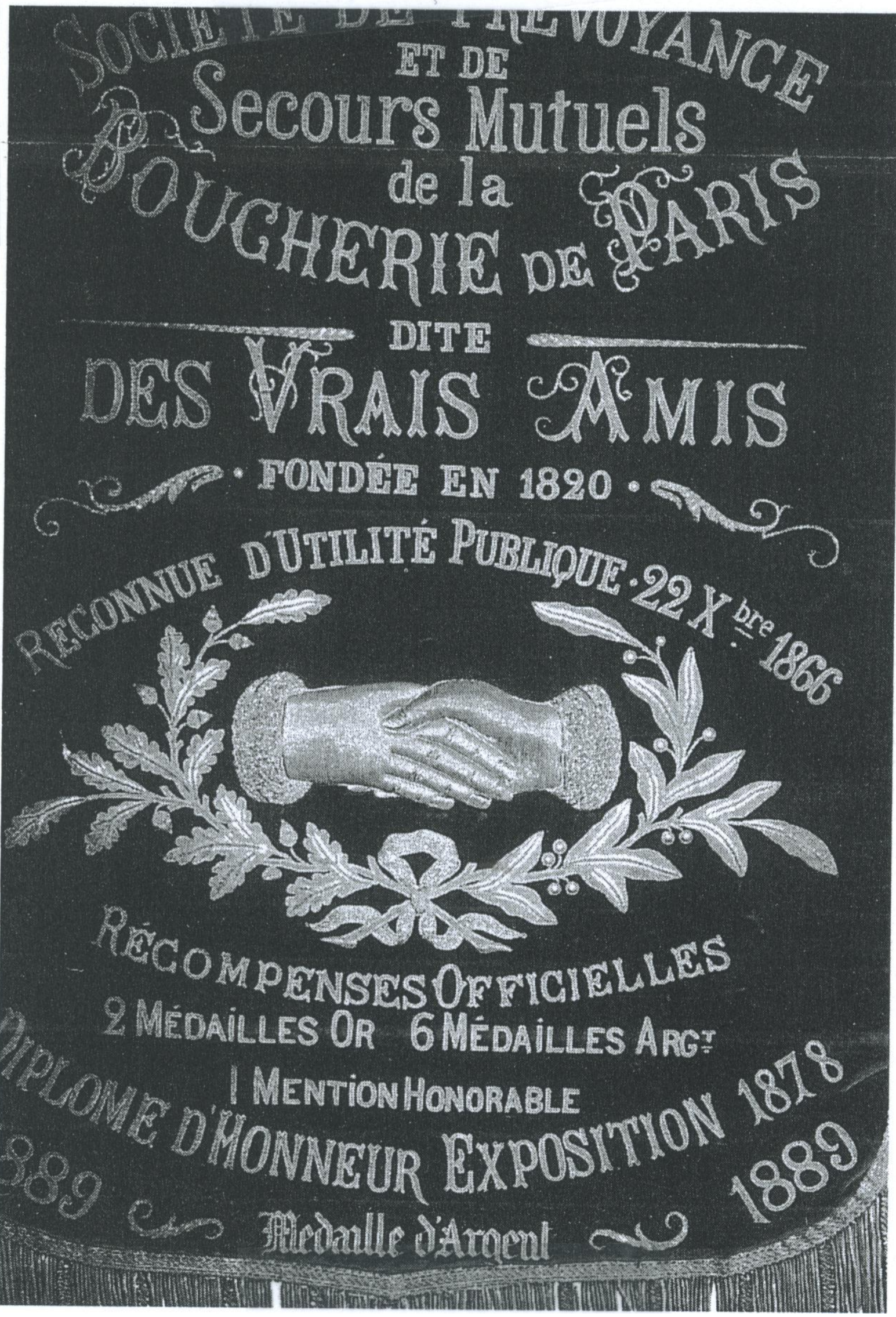

